morbidly obese patients. Interestingly, patients with absent coronary artery calcium had a greater excess weight loss by 12 months (range 6-24) following surgery. Coronary artery calcium scoring may be a useful tool in helping further stratify cardiovascular risk and operative outcomes in bariatric populations.

\section{TRANSFORMING POST TRIAGE ASSESSMENT OF CHEST PAIN IN A BUSY EMERGENCY DEPARTMENT (ED), FROM ED DOCTOR TO ADVANCED NURSE PRACTITIONER (ANP) DIRECT; THE IMPACT ON PATIENT EXPERIENCE TIMES (PET)}

S Ingram. Tallaght University Hospital, Dublin, Ireland

\subsection{6/heartjin-2020-ICS.38}

Background Chest pain accounts for $8 \%$ of TUH Emergency Department (ED) presentations The ANP led chest pain service provides nurse consults to the patient with chest pain in the ED. Once triaged and seen initially by an ED doctor if CHD is suspected the patient is then referred to the ANP nurse led chest pain service for further management.

Patient Experience Time (PET) is the entire time each patient spends in the ED. Since the ANP consult service commenced in 2012, chest pain PET in the ED has reduced from 17 hours, however this time frame is still regularly exceeded with an average PET of 10 hours in 2019. The national ED PET goal is 6 - 9 hours.

Method Building on 6 years of skilled and competent ANP led chest pain consultations in the ED, cardiology ANPs expanded their caseload to assess chest pain patients directly from the waiting room as named clinicians, instead of an ED doctor. The aim was to reduce PET for this patient group by providing focused expert chest pain assessment. Enablers included ANP referral for ionising radiation, ANP medicinal prescribing and buy in from ED Consultants. The ED 'Symphony' system was used as a clinical and audit tool. This initiative commenced in 2017 with 1 ANP annually. All patients seen directly by the ANP since 2017 were included in the data analysis. ANP PET was compared to ED Doctor PET.

Results 200 patients were autonomously managed by the ANPs as named clinicians after initial triage. Over the 3 years average PET for cases managed autonomously by the ANP was 7.5 hours compared with a usual physician care average of 9.5 hours. ANP decision time to admit or discharge was 2.6 hours.

Implications As emergency departments face regular overcrowding, and social distancing now impacts more than ever on available space in the $\mathrm{ED}$, further caseload expansion by the Cardiology ANPs demonstrates further PET reductions in line with targets. Autonomous ANP management frees ED doctors to manage other cases and improves patient flow. Professional courage to work autonomously, delivering safe, evidence-based care with this high-risk group has transformed patient management in this busy ED.

\section{IMPAIRED AUTONOMIC FUNCTION AFTER INCOMPLETE CARDIAC REVASCULARISATION}

R Armstrong, P Wheen, J Cosgrave, RA Kenny, A Maree. St. James's Hospital, Dublin, Ireland

10.1136/heartjnl-2020-ICS.39
Introduction Incomplete cardiac revascularisation (ICR) assessed by residual SYNTAX score ( $\mathrm{rSs}$ ) is associated with increased 5 year mortality. Furthermore, in the general population our group has demonstrated that impaired autonomic function is associated with increased all-cause mortality.

Purpose We hypothesised that ICR would be associated with impaired autonomic function.

Methods After ethical approval and informed consent, consecutive patients attending cardiac rehabilitation in a tertiary referral centre were enrolled. All patients had percutaneous coronary revascularisation. Assessment of autonomic function was performed by determining speed of heart rate recovery between 10 and 20 seconds post orthostatic challenge (HRR10-20). During an active stand, real time heart rate, blood pressure and ECG recordings were taken using non-invasive digital photoplethysmography and HRR10-20 determined. Patients with an $\mathrm{rSs}>0$ were considered incompletely revascularised and those with an $\mathrm{rSs}$ of 0 fully revascularised. Demographic data were recorded and statistical analysis performed (SPSS v23).

Results Patients $(n=40)$ comprised those with complete (CR) $(n=30)$ and incomplete (ICR) $(n=10)$ revascularisation. In the ICR group mean $\mathrm{rSs}$ was 9. HRR10-20 was impaired in the ICR group (mean -2.9) compared to the CR cohort (mean 5.3) $(\mathrm{p}=0.001)$. Completeness of revascularisation was strongly associated with HRR10-20 (Pearson's correlation coefficient $0.529 ; \mathrm{p} \leq 0.0001)$. (Figure 1) Baseline demographics did not differ significantly. Use of rate limiting medication was similar between cohorts (beta blockers, calcium channel blockers, ivabradine).

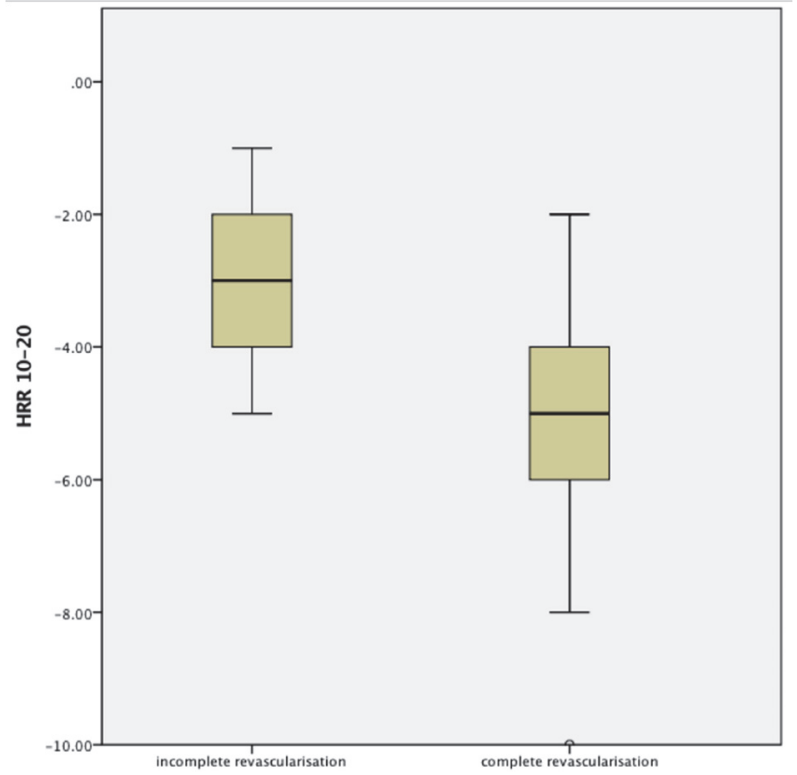

Abstract 39 Figure 1 Results

Conclusions Our data confirm significant correlation between ICR and impaired autonomic function determined by speed of heart rate recovery. Thus determining autonomic dysfunction post ICR may identify those at increased mortality risk. 